\title{
ANALYSIS OF MODIFIED OPTIMIZATION IN MULTIVARIBLE PREDICTIVE CONTROL WITH REGARDS TO CONTROL QUALITY
}

\author{
Marek Kubalcik', Vladimir Bobal'2, Tomas Barot ${ }^{3}$ \\ 1,2 Department of Process Control, Tomas Bata University in Zlin, \\ Faculty of Applied Informatics, Nad Stranemi 4511, 760 05, Zlin, Czech Republic \\ ${ }^{3}$ Department of Mathematics with Didactics, University of Ostrava, \\ Faculty of Education, Frani Sramka 3, 70900 Ostrava, Czech Republic \\ kubalcik@fai.utb.cz, bobal@fai.utb.cz, Tomas.Barot@osu.cz
}

\begin{abstract}
Model predictive control (MPC) has been a widely researched strategy in the modern control theory. Various modifications of MPC are focused on improving of two subsystems - the predictor and the optimizer, which cooperate mutually on the receding horizon. In constrained predictive control, the optimization is a quadratic programming problem, which has to be solved numerically. Namely, in constrained multivariable MPC, the constraints and the multi-variability of the process cause excessive increasing of the computational complexity. Reducing of computational time and decreasing of a number of numerical operations are particularly desirable. This is the reason for efforts to modify algorithms of numerical optimization. These modifications can influence control quality. This paper deals with one modification of the Hildreth optimization method and accurate analysis of its impact to quality of control. For analysis of impact to control quality, descriptive statistical methods are frequently used; particularly, methods based on analysis of floating point operations are complemented with their regression analysis for expression of complexity function $O$. However, the regression analysis of these results belongs only to noninductive statistical methods depending on particular measurements of a computational complexity. For purposes of increasing responsibility of control quality indicators, set of specific statistical methods aimed on testing hypotheses is recommended in this paper. Testing hypotheses can be applied with strictly defined significance level. Practical implementation of modified MPC algorithm is statistically analyzed in comparison with unmodified MPC using proposed set of particular statistical methods.
\end{abstract}

Keywords: Model Predictive Control, Multivariable Process, Optimization, Quadratic Programming, Control Quality, Complexity Function 0, Nonlinear Regression, Testing Hypothesis.

\section{Introduction}

Model predictive control (MPC) [1]-[2] has been widely applied in controlling of industrial processes thanks to its ability to deal with control difficulties such as constrained variables, time-delay and nonlinearity. Predictive control is also one of the most effective approaches for control of multivariable systems [3]. An advantage of model predictive control is that the multivariable systems [4]-[6] can be handled in a straightforward manner.

A structure of a predictive controller can be divided into two parts: a predictor [2] and an optimizer [7]. The basic idea of MPC is to use a model of a controlled process to predict future outputs of the process. A trajectory of future manipulated variables is given by solving an optimization problem [8] incorporating a suitable cost function [8] and constraints [8]. Only the first element of the obtained control sequence is applied. The whole procedure is repeated in following sampling period.
This principle is known as the receding horizon strategy [1].

A significantly important part of the model predictive control with constraints [2] are algorithms of numerical optimization [9]. Reduction of the computational complexity [10] of the optimization methods has been widely researched [11]-[12]. The Hildreth's method [13, p. 67-68] based on the dual-problem-optimization-principles [6] has been widely applied and implemented in the model predictive control. However, modifications of this method are not widely described in context of the model predictive control.

One possible modification of the Hildreth method, which reduces the computational complexity of the algorithm, and its application in the multivariable predictive control was presented in [14]. The main aim of this paper is to propose a detailed statistical analysis of influence of the modification of the control algorithm on control quality. Simulation results of control with 
implemented modification in the optimization part of the predictive controller are shown in this paper. The analysis quantifies improvement of the computational efficiency when using the applied modification. A set of statistical methods based on mathematical induction for analysis of control quality was proposed.

\section{Multivariable Model Predictive Control}

Further a system with two inputs and two outputs will be considered. The two - input/two - output (TITO) processes are the most often encountered multivariable processes in practice and many processes with inputs/outputs beyond two can be treated as several TITO subsystems [3].

A mathematical model of TITO process can be considered in the form of matrix fraction

$$
\boldsymbol{G}\left(z^{-1}\right)=\boldsymbol{A}^{-1}\left(z^{-1}\right) \boldsymbol{B}\left(z^{-1}\right)
$$

This model can be transcribed to difference equations [3] which can be used for computation of the systems output predictions [2] over minimum $N_{1}$ and maximum $N_{2}$ horizons. Further the polynomials in the matrices $\boldsymbol{A}$ and $\boldsymbol{B}$ will be considered to be of the second order. Further we will consider constraints of the manipulated variables. In a constrained case, a vector of future increments of the manipulated variables is given by solving an optimization problem given by (2), where $\boldsymbol{y}, \boldsymbol{u}$, and $\boldsymbol{w}$ are vectors of controlled, manipulated and reference signals. The matrices $\boldsymbol{I}, \boldsymbol{P}, \boldsymbol{G}$ leads from the prediction equations [2].

$$
\left.\begin{array}{c}
\Delta \boldsymbol{u}=\arg \min \left\{\frac{1}{2} \Delta \boldsymbol{u}^{T} \boldsymbol{H} \Delta \boldsymbol{u}+\boldsymbol{b}^{T} \Delta \boldsymbol{u} \mid \boldsymbol{M} \Delta \boldsymbol{u} \leq \boldsymbol{\gamma}\right\} \\
\boldsymbol{H}=\boldsymbol{G}^{T} \boldsymbol{G}+\boldsymbol{I} ; \boldsymbol{b}=\boldsymbol{G}^{\boldsymbol{T}}\left(\boldsymbol{P}\left[\begin{array}{c}
\boldsymbol{y}(k) \\
\boldsymbol{y}(k-1) \\
\boldsymbol{y}(k-2) \\
\Delta \boldsymbol{u}(k-1)
\end{array}\right]-\boldsymbol{w}\right)
\end{array}\right\}
$$

Only the first element of the obtained control sequence $\boldsymbol{\Delta} \boldsymbol{u}$ is applied. The vector $\boldsymbol{\Delta} \boldsymbol{u}$ has a length that corresponds to the control horizon $N_{\mathrm{u}}$.

\section{Optimization Strategies in Model Predictive Control}

Optimization problem (2) [7] is then solved numerically by quadratic programming in each sampling period. In practical applications of the multivariable MPC, the Hildreth's method is frequently applied for the quadratic programming optimization solution, as is it was recommended in [13]. The Hildreth's method is based on numerical iterations, in which particular subresults are gradually improving. The method can be categorized as a dual optimization method [8].
The first setting of dual vector variable (3) can be considered in form of a multidimensional extreme [9] without any constraints. The dual solution (3), which is further directly applied into the current step of the discrete MPC, is transformed into the form of the primary solution [9] (5)

$$
\begin{aligned}
& \boldsymbol{d} \in \mathbb{R}^{N u, 1} ; \boldsymbol{d}=\left[\begin{array}{lll}
d_{1} & \cdots & d_{N u}
\end{array}\right]^{T}= \\
& =\arg \min \left\{\frac{1}{2} \boldsymbol{d}^{T} \boldsymbol{N} \boldsymbol{d}+\boldsymbol{o}^{T} \boldsymbol{d} \mid \boldsymbol{d} \geq \boldsymbol{0}\right\} ; \\
& \left.\begin{array}{l}
\boldsymbol{N}=\boldsymbol{M} \boldsymbol{H}^{-1} \boldsymbol{M}^{T} ; N_{i j} \in \boldsymbol{N} \in \mathbb{R}^{N u, N u} ; \\
\boldsymbol{o}=\boldsymbol{M} \boldsymbol{H}^{-1} \boldsymbol{b}^{T}+\boldsymbol{\gamma} ; o_{i j} \in \boldsymbol{O} \in \mathbb{R}^{N u, 1}
\end{array}\right\} \\
& \boldsymbol{\Delta u}(k)=-\boldsymbol{H}^{-1}\left(\boldsymbol{M}^{T} \boldsymbol{d}+\boldsymbol{b}^{T}\right)
\end{aligned}
$$

In the Hildreth's method, the first computation of dual variable (3) is further improved in the iteration based cycle [13, p. 67-68]. An index of iteration is denoted as $\omega$ and the improved solution of the dual variable is defined by (6).

$$
\boldsymbol{d}(\omega)=\left[\begin{array}{lll}
d_{1}(\omega) & \cdots & d_{N u}(\omega)
\end{array}\right]^{T}
$$

As MPC has specific forms of matrices in the definition of the optimization problem, the multidimensional extreme does not occur frequently. For the constrained manipulated variable, the matrices $\boldsymbol{M}$ and $\boldsymbol{\gamma}$ have a form (7), where $\boldsymbol{T}$ is a lower triangular matrix.

$$
\begin{aligned}
& \boldsymbol{M}=\left[\begin{array}{c}
-\boldsymbol{T} \\
\boldsymbol{T}
\end{array}\right] \\
& \left.\boldsymbol{\gamma}=\left[\begin{array}{lll}
{\left[u(k-1)-u_{\min }\right.} & \cdots & \left.u(k-1)-u_{\min }\right]^{T} \\
{\left[u_{\max }-u(k-1)\right.} & \cdots & \left.u_{\max }-u(k-1)\right]^{T}
\end{array}\right]\right\}
\end{aligned}
$$

The computational complexity of the optimization problem of the quadratic programming (2) is significantly increased by constraints in the MPC and by higher values of the horizons. In case of the multivariable control, the decreasing of the algorithm-complexity is particularly important.

In [11], an explicit approach was presented which is based on off-line pre-computed optimal solutions. Some other researches has tried to modify the numerical method itself e.g. in the algorithms of the Sequence Linear Programming [12] or Interior Point Methods [11]. In this paper, a modification of the dual Hildreth's method [13], which is recommended for using in multivariable predictive control [14] is applied. The applied modification is based on an addition of a new condition (8).

$$
\boldsymbol{M}\left[-\boldsymbol{H}^{-1}\left(\boldsymbol{M}^{T} \boldsymbol{d}(\omega)+\boldsymbol{b}^{T}\right)\right] \leq \boldsymbol{\gamma}
$$

Equation (8) was than transformed to dual problem (9) in the iterative algorithm in the computational cycle of the algorithm.

$$
\boldsymbol{d}(\omega) \geq\left[\begin{array}{lll}
0 & \cdots & 0
\end{array}\right]^{T} ; \boldsymbol{d}(\omega) \in \mathbb{R}^{N u, 1}
$$




\section{Control Quality of Model Predictive Control}

\subsection{Generally used assessment methods}

The aim was also assessment of control quality with and without the proposed modification. Changes in numbers and floating point operations [10] were analysed before and after implementation of the modification.

Generally used nonlinear regression [15] can be advantageous for determination of complexity function $O=O(n)$ [10]. This construction is based on numbers of floating point operations depending on a problem size $n$, which is increasing. However, this regression analysis belongs to a class of only descriptive statistical methods [16].

Complexity function $O$ can be expressed from this regression analysis as the first part of regression equation $F=F(n)$. In the complexity function $O$, the first part can be defined as an element of the regression equation with the highest degree multiplied by a constant.

Second possibility is measurement of control quality criterions [14]:

$$
\begin{gathered}
J_{1}=\sum_{k}\left[\Delta u_{1}(k)\right]^{2}+\sum_{k}\left[\Delta u_{2}(k)\right]^{2} \\
J_{2}=\sum_{k}\left[w_{1}(k)-y_{1}(k)\right]^{2}+\sum_{k}\left[w_{2}(k)-y_{2}(k)\right]^{2}
\end{gathered}
$$

\subsection{Proposal of set of methods applicable in assessment of control quality}

In this paper, a set of more accurate methods for control quality assessment is proposed.

Statistical methods which include testing hypotheses are potentially advantageous for accurate testing of control quality by the measured criterions (10)-(11). For purposes of technical application, it is suitable the strictly defined significance level $\alpha$ equal to 0.001 .

For assessment of control quality, application of testing hypotheses on existence of statistical significant differences before and after realized modifications can be appropriate. In each pair of measured values of criterions, this testing hypothesis can be performed.

Depending on properties of measured criterions (10)-(11) before and after applied modifications in control algorithm, a type of a particular method for testing of statistically significant differences between the considered pairs of the criterion values should be selected. For a case with fulfilling normality [17] of data, parametric method paired T-test [16] should by applied on these data. In case when normality is not fulfilled, the non-parametric Wilcoxon test [16] should be alternatively used. These properties of data based on normality can be tested using the normality tests Shapiro-Wilk [17] and AndersonDarling [17] on the same significance level $\alpha$ considered in the whole assessment of control quality. Both paired T-test and Wilcoxon tests are based on the same assumptions defined by zero hypothesis (12) and alternative hypothesis (13), where parameter $\Delta$ is mean value for parametric test [16]-[18] and median for non-parametric test [16][18].

$$
\begin{aligned}
& H_{0}: \Delta_{1}=\Delta_{2} \\
& H_{1}: \Delta_{1} \neq \Delta_{2}
\end{aligned}
$$

Conclusions of testing of normality and testing of statistically significant differences are based on comparison of result of testing of each hypothesis in form of $p$-value with considered significance level $\alpha$. In case of $p \geq \alpha$, the zero hypothesis is failed to reject on the significance level $\alpha$ and this situation implicates a conclusion, that there are not statistically significant differences between pairs of compared data. In the opposite case, the zero hypothesis is rejected in favor of alternative hypothesis, which indicates existence of statistically significant differences between pairs of measured data on the considered significance level $\alpha$.

\section{Results}

\subsection{Constrained multivariable predictive control}

The modifications [14] of standard optimization techniques were applied in MPC of multivariable process defined by following polynomial matrices (14)-(15).

$$
\left.\begin{array}{l}
\boldsymbol{A}\left(z^{-1}\right)=\left[\begin{array}{ll}
A_{11}\left(z^{-1}\right) & A_{12}\left(z^{-1}\right) \\
A_{21}\left(z^{-1}\right) & A_{22}\left(z^{-1}\right)
\end{array}\right] \\
A_{11}\left(z^{-1}\right)=1-1.32640 z^{-1}+0.3271 z^{-2} \\
A_{12}\left(z^{-1}\right)=0.0240 z^{-1}-0.0029 z^{-2} \\
A_{21}\left(z^{-1}\right)=-0.0711 z^{-1}+0.0759 z^{-2} \\
A_{22}\left(z^{-1}\right)=1-1.0911 z^{-1}+0.1340 z^{-2}
\end{array}\right\}
$$




$$
\left.\begin{array}{l}
\boldsymbol{B}\left(z^{-1}\right)=\left[\begin{array}{ll}
B_{11}\left(z^{-1}\right) & B_{12}\left(z^{-1}\right) \\
B_{21}\left(z^{-1}\right) & B_{22}\left(z^{-1}\right)
\end{array}\right] \\
B_{11}\left(z^{-1}\right)=0.2983 z^{-1}-0.0970 z^{-2} \\
B_{12}\left(z^{-1}\right)=0.0930 z^{-1}+0.0682 z^{-2} \\
B_{21}\left(z^{-1}\right)=0.1755 z^{-1}+0.0688 z^{-2} \\
B_{22}\left(z^{-1}\right)=0.1779 z^{-1}+0.1065 z^{-2}
\end{array}\right\}
$$

Consideration of constraints $u_{\min }=-2, u_{\max }=2$ was reflected in matrix $\boldsymbol{M}$ and in vector $\gamma$, as can be seen in (16).

$$
\begin{aligned}
& M=\left[\begin{array}{c}
-\boldsymbol{T} \\
\boldsymbol{T}
\end{array}\right] \\
& \left.\boldsymbol{\gamma}=\left[\begin{array}{lll}
{[u(k-1)+2} & \cdots & u(k-1)+2]^{T} \\
{[2-u(k-1)} & \cdots & 2-u(k-1)]^{T}
\end{array}\right]\right\}
\end{aligned}
$$

Constrained MPC of two-input and two-output process (14)-(15) was simulated in MATLAB. Courses of control variables achieved during control without the modification can be seen in Fig. 1 and courses of control variables with the modification in Fig. 2.

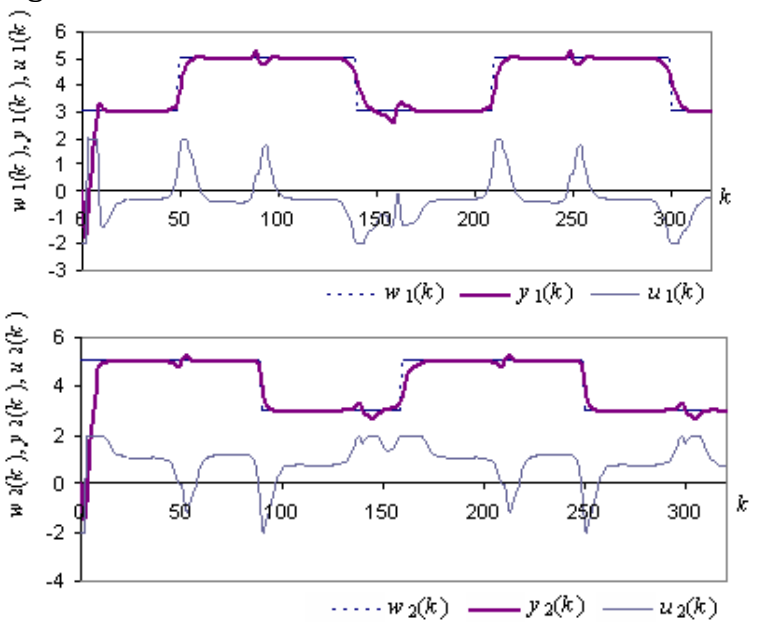

Figure 1: Realized MPC without Modifications

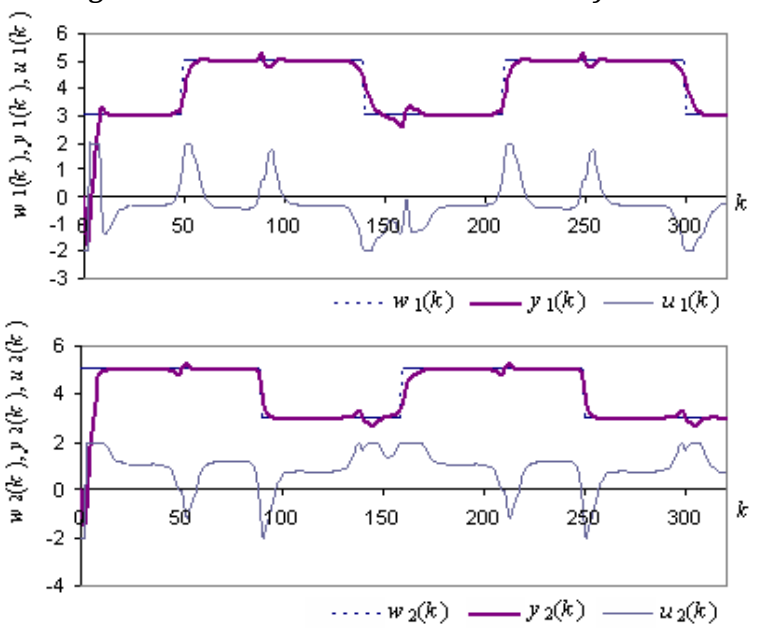

Figure 2: Realized MPC using Modifications

\subsection{Analysis of algorithm complexity using descriptive statistical methods}

For analysis of the computational complexity, methods of descriptive statistics [16], particularly the nonlinear regression analysis [15], were properly used. Evaluation of the complexity was based on dependences between size $\mu$ of the algorithm problem (17) and the computational complexity $O=O(\mu)$ [10], which can be deducted from the regression equation $F=F(\mu)[15]$, where $F$ is in flops [10]. Variable $\mu$ (17) was defined as equal to the control and prediction horizons $N_{u}$ and $N_{2}$. The results of the complexity function were denoted as $O^{*}=O^{*}(\mu)$. The regression equation was denoted as $F^{*}=F^{*}(\mu)$.

$$
\mu=N_{u}=N_{2}=\{5 ; 10 ; 15 ; 20 ; \ldots ; 45\}
$$

Decreasing of the computational complexity is obvious from the results in Table 1 and in Fig. 3.

Table 1. Control Quality and Complexity in MPC and in Modified MPC

\begin{tabular}{|l|l|l|}
\hline$\mu$ & $F$ flops] & \multicolumn{1}{|c|}{$F^{*}$ [flops] } \\
\hline 5 & $2 \times 10^{7}$ & $1 \times 10^{7}$ \\
\hline 10 & $2 \times 10^{8}$ & $1 \times 10^{8}$ \\
\hline 15 & $8 \times 10^{8}$ & $4 \times 10^{8}$ \\
\hline 20 & $2 \times 10^{9}$ & $1 \times 10^{9}$ \\
\hline 25 & $5 \times 10^{9}$ & $2 \times 10^{9}$ \\
\hline 30 & $1 \times 10^{10}$ & $4 \times 10^{9}$ \\
\hline 35 & $2 \times 10^{10}$ & $7 \times 10^{9}$ \\
\hline 40 & $3 \times 10^{10}$ & $1 \times 10^{10}$ \\
\hline 45 & $4 \times 10^{10}$ & $2 \times 10^{10}$ \\
\hline
\end{tabular}

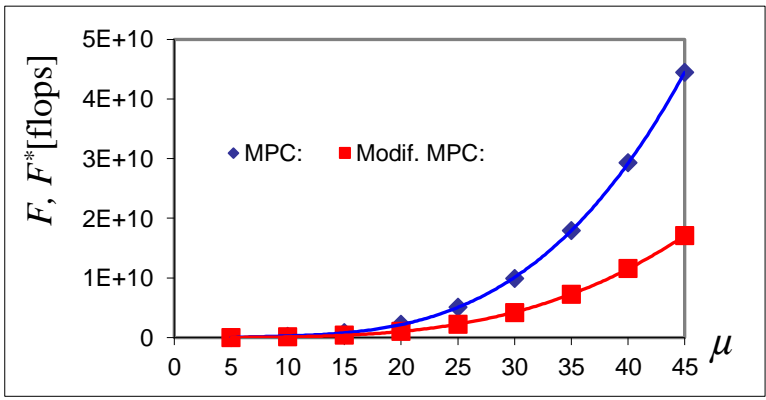

Figure 3: Comparison of Number of Operations using Nonlinear Regression in Realized MPC

The results of control both with and without the applied modification are similar as can be seen in Fig. 1 and Fig. 2. A decreasing of the algorithm computational complexity can be statistically expressed using nonlinear regression analysis in (18) and complexity functions (19). 


$$
\left.\begin{array}{c}
F=854310 \mu^{3}-2 \times 10^{7} \mu^{2}+ \\
+2 \times 10^{8} \mu-7 \times 10^{8} \\
F^{*}=276494 \mu^{3}-5 \times 10^{6} \mu^{2}+ \\
+5 \times 10^{7} \mu-1 \times 10^{8} \\
O=854310 \mu^{3} \\
O^{*}=276494 \mu^{3}
\end{array}\right\}
$$

\subsection{Analysis of algorithm complexity using testing hypotheses with regards to quantifiers of control quality}

Except of the previously presented descriptive statistical procedures, the simulation results of control were also analysed using testing hypotheses with regards to criterions of control quality (10)(11), as it is described in Table 2.

Table 2. Control Quality in MPC and in Modified MPC

\begin{tabular}{|l|l|l|l|l|}
\hline \multirow{2}{*}{$\mu$} & $J_{1}$ & $J_{2}$ & $J_{1}$ & $J_{2}$ \\
\cline { 2 - 5 } & MPC & MPC & $\begin{array}{l}\text { Modified } \\
\text { MPC }\end{array}$ & $\begin{array}{l}\text { Modified } \\
\text { MPC }\end{array}$ \\
\hline 5 & 92.3328 & 268.4459 & 92.3334 & 268.4449 \\
\hline 10 & 88.4763 & 262.1586 & 88.4768 & 262.1578 \\
\hline 15 & 87.6638 & 261.5773 & 87.6642 & 261.5767 \\
\hline 20 & 87.5846 & 261.5629 & 87.585 & 261.5623 \\
\hline 25 & 87.5877 & 261.542 & 87.588 & 261.5415 \\
\hline 30 & 87.6066 & 261.4577 & 87.6068 & 261.4572 \\
\hline 35 & 87.6489 & 261.3348 & 87.649 & 261.3342 \\
\hline 40 & 87.7746 & 261.1032 & 87.7747 & 261.1026 \\
\hline 45 & 88.102 & 260.6048 & 88.1021 & 260.6042 \\
\hline
\end{tabular}

In Table 2, obtained results of the control quality criterions differ on the $3^{\text {rd }}$ decimal place. It is then obvious that the quality of control was not significantly affected by application of the modification in the optimization algorithm.

In order to better quantify contribution of the applied modifications, a set of statistical methods based on mathematical induction was applied with results in $p$-values [16]-[17].

The first step was to verify the normality [17] of the measured values of criterions (10)-(11). The obtained results were tested using the Shapiro-Wilk and Anderson-Darling test on $\alpha$ equal to 0.001 . The results of testing normality are displayed in Table 3. Results in form of $p$-values were obtained using software PAST Statistics [19]. Unfulfilling of normality of data was identified by comparison of $p$ values with $\alpha$.
Table 3. Testing Normality of Data Set of Quantifiers

\begin{tabular}{|l|l|l|l|}
\hline$J_{1}$ & $J_{2}$ & $J_{1}$ & $J_{2}$ \\
\hline MPC & MPC & $\begin{array}{l}\text { Modif. } \\
\text { MPC }\end{array}$ & $\begin{array}{l}\text { Modif. } \\
\text { MPC }\end{array}$ \\
\hline$p$-values Obtained Using Shapiro-Wilk Test \\
\hline $1.81 \times 10^{-5}$ & $1.81 \times 10^{-5}$ & $2.88 \times 10^{-5}$ & $2.88 \times 10^{-5}$ \\
\hline$p$-values Obtained Using Anderson-Darling Test \\
\hline $2.59 \times 10^{-5}$ & $2.59 \times 10^{-5}$ & $2.80 \times 10^{-5}$ & $2.80 \times 10^{-5}$ \\
\hline
\end{tabular}

The second step was testing hypotheses on existence of statistically significant differences in sets of pairs of data on the significance level $\alpha$ equal to 0.001 .

The values of criterions from Table 1 were tested using the non-parametric exact Wilcoxon paired test [16] with regards to unfulfilling normality of data (Table 3). This test can confirm an existence of statistically significant differences in data set with paired based data by comparing medians defined as zero and alternative hypothesis (20)-(21). Where zero hypothesis (20) can be expressed as nonexistence of statistically significant differences between pairs of data of particular criterion $\left(J_{1}\right.$, respectively $J_{2}$ ). Alternative hypothesis (21) is based on testing the existence of these differences.

$$
\begin{aligned}
& H_{0}: \tilde{x}_{1}=\tilde{x}_{2} \\
& H_{1}: \tilde{x}_{1} \neq \tilde{x}_{2}
\end{aligned}
$$

By using software PAST Statistics [19], results of testing hypotheses (20)-(21) (Table 4) in form of $p$ values were achieved and then compared with the significance value $\alpha$.

Table 4. Testing Hypotheses on Data Set of Quantifiers

\begin{tabular}{|l|l|l|l|}
\hline$J_{1}$ & $J_{1}$ & $J_{2}$ & $J_{2}$ \\
\hline MPC & $\begin{array}{l}\text { Modif. } \\
\text { MPC }\end{array}$ & MPC & $\begin{array}{l}\text { Modif. } \\
\text { MPC }\end{array}$ \\
\hline $\begin{array}{l}p \text {-value: } 0.005848> \\
>(\alpha=0.001)\end{array}$ & $\begin{array}{l}p \text {-value: } 0.00377> \\
>(\alpha=0.001)\end{array}$ \\
\hline
\end{tabular}

As can be seen in Table 4, for both criterions, non-existence of statistically significant differences was indicated on the significance level 0.001. Failing to rejection of all proposed hypotheses proved that the application of the modification in the optimization part of MPC did not significantly influence the quality of the control.

\section{Conclusions}

The modifications of standard optimization techniques were applied in multivariable MPC with constraints. Saving of computational time was then achieved. It was proved by nonlinear regression analysis, where decreasing of number of floating point operations was indicated. The methods of nonlinear regression analysis belong to a class of descriptive statistical methods. 
In order to better quantify contribution of the applied modifications, the simulation results of control were also analysed using testing hypotheses with regards to control quality. A set of statistical methods based on mathematical induction was proposed in this paper, which can bring more accurate quantification of the influences of changes in control algorithm on control quality.

\section{References}

[1] Kwon WH. Receding horizon control: model predictive control for state models. Springer, 2005.

[2] Corriou JP. Process control: theory and applications. Springer, 2004.

[3] Navratil P., Pekar L. Possible approach to control of multi-variable control loop by using tools for determining optimal control pairs. International Journal of Circuits, Systems and Signal Processing, 2013, 7(6): 91-96.

[4] Daniel B., Octavian D., Gheorghe GI., Constantin V., Alina S. Mechatronic system for solar energy acquisition. International Journal of Mechatronics and Applied Mechanics, 2017, 2017(1): pp. 75-80.

[5] Krayem S., Patikova, Z. An insight into DVB-T system using formal modelling in Event-B. AIP Conference Proceedings, 2017, 1863.

[6] Daniel CC., Mihail MA., Viorel G., Cristinel I. Modelling and simulation for control of a mems electromagnetic scanner. International Journal of Mechatronics and Applied Mechanics, 2017, 2017(2): pp. 7-14.

[7] Lee GM., Tam NN., Yen ND. Quadratic Programming and Affine Variational Inequalities: A Qualitative Study. Springer, 2005.

[8] Dostal Z. Optimal Quadratic Programming Algorithms: With Applications to Variational Inequalities. Springer, 2009.

[9] Luenberger D.G., Ye Y. Linear and nonlinear programming. Springer, 2008.
[10] Hunger R. Floating Point Operations in MatrixVector Calculus. (Version 1.3). Technical Report. Technische Universität München, Associate Institute for Signal Processing, 2007. https:// mediatum.ub.tum.de/doc/625604/625604.pdf. accessed at:22.11.2018

[11] Hertog D. Interior point approach to linear, quadratic, and convex programming: algorithms and complexity. Kluwer Academic Publishers, 1994.

[12] Rao SS. Engineering optimization: theory and practice. 4th ed. Hoboken, 2009.

[13] Wang L. Model Predictive Control System Design and Implementation Using MATLAB. Springer, 2009.

[14] Kubalcik M., Bobal V., Barot T. Modified Hildreth's Method Applied in Multivariable Model Predictive Control. HELIX 2018: Innovation, Engineering and Entrepreneurship. Lecture Notes in Electrical Engineering, 2019, 505: 75-81.

[15] Krivy I., Tvrdik J., Krpec R. Stochastic algorithms in nonlinear regression. Computational Statistics and Data Analysis, vol. 33(12), pp. 277-290. Elsevier, 2000.

[16] Kitchenham B., Madeyski L., Budgen D., et al. Robust Statistical Methods for Empirical Software Engineering. Empirical Software Engineering, 2016: 1-52.

[17] Vaclavik M., Sikorova Z., Barot T. Particular Analysis of Normality of Data in Applied Quantitative Research. 2nd Computational Methods in Systems and Software 2018: Computational and Statistical Methods in Intelligent Systems. Advances in Intelligent Systems and Computing, 2019, 859: 353-365.

[18] Sulovska K., Belaskova S., Adamek M. Gait patterns for crime fighting: Statistical evaluation. Proceedings of SPIE - The International Society for Optical Engineering, 2013, 8901.

[19] Hammer O., Harper DAT., Ryan PD. PAST: Paleontological statistics software package for education and data analysis. Palaeontologia Electronica, 2001, 4(1). 